\title{
L'ORGANISATION DE LA PRATIQUE DU CANYONING SUR UN SITE : LE CANYON DU FURON
}

\author{
Clémence Perrin et Jean-Pierre Mounet
}

EDP Sciences | Movement \& Sport Sciences

\author{
2006/1 - no 57 \\ pages 79 à 103
}

\section{ISSN 1378-1863}

Article disponible en ligne à l'adresse:

http://www.cairn.info/revue-science-et-motricite-2006-1-page-79.htm

Pour citer cet article :

Perrin Clémence et Mounet Jean-Pierre, « L'organisation de la pratique du canyoning sur un site : le canyon du Furon »,

Movement \& Sport Sciences, 2006/1 no 57, p. 79-103. DOI : 10.3917/sm.057.103

Distribution électronique Cairn.info pour EDP Sciences.

(c) EDP Sciences. Tous droits réservés pour tous pays.

La reproduction ou représentation de cet article, notamment par photocopie, n'est autorisée que dans les limites des conditions générales d'utilisation du site ou, le cas échéant, des conditions générales de la licence souscrite par votre établissement. Toute autre reproduction ou représentation, en tout ou partie, sous quelque forme et de quelque manière que ce soit, est interdite sauf accord préalable et écrit de l'éditeur, en dehors des cas prévus par la législation en vigueur en France. II est précisé que son stockage dans une base de données est également interdit. 


\title{
L'organisation de la pratique du canyoning sur un site : le canyon du Furon
}

\section{De Boeck Université | Science et motricité}

\author{
2006/1 - no 57 \\ pages 79 à 103 \\ ISSN 1378-1863
}

Article disponible en ligne à l'adresse:

http://www.cairn.info/revue-science-et-motricite-2006-1-page-79.htm

\section{Pour citer cet article :}

"L'organisation de la pratique du canyoning sur un site : le canyon du Furon", Science et motricité, 2006/1 no 57, p. 79-103.

Distribution électronique Cairn.info pour De Boeck Université.

() De Boeck Université. Tous droits réservés pour tous pays.

La reproduction ou représentation de cet article, notamment par photocopie, n'est autorisée que dans les limites des conditions générales d'utilisation du site ou, le cas échéant, des conditions générales de la licence souscrite par votre établissement. Toute autre reproduction ou représentation, en tout ou partie, sous quelque forme et de quelque manière que ce soit, est interdite sauf accord préalable et écrit de l'éditeur, en dehors des cas prévus par la législation en vigueur en France. II est précisé que son stockage dans une base de données est également interdit. 


\title{
L'organisation de la pratique du canyoning sur un site : le canyon du Furon
}

\author{
Clémence Perrin et Jean-Pierre Mounet ${ }^{(1)}$
}

\section{RÉSUMÉ}

Depuis les années 1980, la pratique des sports de nature augmente dans les Parcs naturels régionaux, amenant leurs gestionnaires à s'interroger sur le développement de ces activités. Cette étude s'intéresse à la pratique du canyoning dans le Parc naturel régional du Vercors. En utilisant les concepts développés par Friedberg (1993) pour l'analyse de l'action organisée, il s'agit de comprendre comment s'organise cette activité sur un site de pratique: le canyon du Furon. Sur ce site, la présence d'un barrage EDF pèse fortement sur la sécurité des personnes engagées dans le canyon. L'accès au canyon s'est donc révélé être un problème pour les utilisateurs. Pour le résoudre, les acteurs concernés par ce problème ont été amenés à coopérer. Cette coopération a abouti à la signature de conventions entre EDF et les fédérations concernées, permettant la stabilisation des relations entre les acteurs. Toutefois, ces relations restent sous-tendues par des enjeux et des intérêts En définitive, les résultats mettent en évidence la difficile gestion d'une activité sportive de nature telle que le canyoning.

Mots-clés : canyoning, action organisée, ordre local.

(1) Laboratoire SENS (Sport et environnement social), UFR APS de l'Université Joseph Fourier, Grenoble 1. Correspondance : Clémence Perrin, UFR APS, BP 43, 38041 Grenoble Cedex 9 - Tél. : 0476514208 - clemence.perrin@ujf-grenoble.fr. 


\title{
The sporting organization of canyoneering at one location: the Furon canyon
}

\begin{abstract}
An increase in the practice of nature sports in the Regional Parks since the eighties has led the park authorities to enquire into how these activities develop. The present study focuses on the practice of canyoneering in the Regional Park of the Vercors. Using the concepts developed by Friedberg (1993) for the analysis of organised action, the aim is to understand how this activity is organised in one location: the Furon Canyon. In this location, an upstream hydroelectric dam compromises the safety of people situated in the canyon. Therefore, access to the canyon turned out to be a problem for the users. In order to solve the problem, the agents concerned were forced to cooperate. This cooperation has led to an agreement between the French utilities Agency and the affected associations that allowed the relationship between the agents to stabilise. Nevertheless, this relationship remains in a tension between different stakes and interests. Ultimately, results highlight the difficult management of a nature sport such as the canyoneering.
\end{abstract}

Key words: canyoneering, organized action, local order.

\section{Introduction}

Durant les quarante dernières années, le taux de pratique sportive a fortement progressé en France. Selon les différentes enquêtes (Guarrigues, 1988 ; Irlinger, Louveau \& Métoudi, 1987 ; Pouquet, 1994), le nombre de Français s'adonnant au sport se situe entre $43 \%$ et $73 \%$, en fonction de la définition du sport adoptée par les chercheurs. Mais la tendance qui se dégage le plus nettement est un engouement pour des activités sportives qui se pratiquent dans les grands espaces libres, incertains ou inconnus de la nature. En effet, les citadins sont à la recherche de nouveaux cadres d'exercices, proches de situations naturelles : ils délaissent les équipements traditionnels et migrent vers les espaces écologiques de la périphérie (Pociello, 1995). La nature exerce sur eux un attrait car elle est « un support imaginaire de la liberté et un gisement jugé inépuisable d'espaces à traverser, de milieux à conquérir et de sources d'énergies à exploiter » (Pociello, 1995, p. 261). Ainsi les Parcs Naturels Régionaux (PNR) qui sont des territoires de nature par excellence présentent « un attrait tout particulier pour les touristes ou les sportifs qui y voient un terrain privilégié pour des pratiques originales et des sensations nouvelles » (Peseux, Sagart, Mounet \& Delaye, 1999, p. 5). Or les PNR, qui ont comme vocation la préservation de l'environnement et le développement économi- 
que local, sont amenés à s'interroger sur le développement de ces pratiques et sur leur insertion parmi les autres activités humaines. Certains Parcs ont déjà engagé des actions sur leur territoire dans le but de connaître et d'organiser les différentes pratiques. Il convient de préciser que les PNR ont un statut de collectivités territoriales et ne disposent pas de pouvoir réglementaire sur l'espace. Leurs moyens d'action relèvent davantage de l'information et de l'animation auprès des acteurs locaux (Fédération des Parcs naturels régionaux de France, 2000).

En Rhône-Alpes, le Parc naturel régional du Vercors (PNRV) s'implique dans la gestion des sports de nature. Cette implication est précisée dans sa charte comme " un enjeu majeur relevant d'un tourisme de qualité (conforter l'offre et diversifier le produit touristique)». Les actions du Parc sont cadrées dans un schéma d'organisation des sports de nature. Afin de pouvoir rédiger ces schémas, il est nécessaire de savoir comment s'organisent les activités sur les sites les plus fréquentés. Ce travail se situe donc dans le cadre d'une convention de recherche signée avec le PNRV et concerne la pratique du canyoning. Son objectif est de comprendre comment est organisée cette activité sur l'un des sites de pratique les plus fréquentés du Vercors : le canyon du Furon.

\section{Le développement de l'activité canyoning en France}

Le mot « canyon » vient de l'espagnol « cañon » qui signifie tuyau, tube. Le canyoning ou descente de canyon est " une pratique qui consiste à descendre le lit d'une rivière encaissée en faisant appel à différentes disciplines comme l'escalade, la natation (souvent en eau vive) et la marche, et qui nécessite le recours à l'utilisation d'agrès » (Annexe canyon de l'arrêté ministériel du 8 décembre 1995).

À l'origine, l'activité canyoning consiste en une exploration des canyons par des spéléologues et des guides, notamment dans les Pyrénées. Dans les années 1970, la pratique se développe dans la Sierra de Guara et dans les Alpes Maritimes où « quelques spéléologues délaissent momentanément grottes et rivières souterraines pour explorer les clues de la région en utilisant leurs techniques de spéléologie à ciel ouvert » (Alexis, 1997, p. 3). Puis, à partir de 1975, la descente de canyon comme pratique collective prend son essor. Les premiers professionnels occasionnels encadrent l'activité dans la Sierra de Guara, puis sur des sites français : "Accompagnateurs de moyenne montagne, indépendants ou cadres fédéraux des fédérations de Montagne et de Spéléologie sont employés dans de pseudo-associations. L'absence de réglementation spécifique 
laisse place à la liberté d'œuvrer dans un flou juridique relativement permissif » (Alexis, 1997, p. 4). Dans ce contexte, de nombreux guides, professionnels de la Spéléologie ou du Kayak, font du canyoning une activité à part entière pendant les mois d'été.

Depuis les années 1980, les flux de pratiquants dans les canyons sont de plus en plus importants. Même s'il n'existe aucune statistique officielle du nombre de pratiquants, plusieurs données sont disponibles pour connaître la fréquentation de certains sites. La fréquentation annuelle du canyon du Llech dans le massif du Canigou est de l'ordre de sept à dix mille personnes (Section permanente Prévention/Information du CSSM, 1996). Quatre à six mille personnes fréquentent les gorges du Tapoul dans les Cévennes en juillet-août (Rigolet, 1995). En 1997, le canyon de l'Eau Rousse en Tarentaise a connu 6000 passages de personnes encadrées par des guides de haute montagne (Agence touristique départementale de la Savoie, 1997). En juillet-août 2000, plus de 5000 personnes ont fréquenté les canyons des Écouges et du Furon dans le Vercors (Boudières, 2000). Enfin, l'Union des Centres de Plein Air (UCPA) comptabilise chaque année environ 10000 journées stagiaires.

Le développement du canyoning a suscité l'intérêt de plusieurs fédérations sportives. La Fédération Française de Spéléologie (FFS) a été la première à s'intéresser à cette activité. Le 18 juin 1986 est créée, au sein de la fédération, la délégation spéléologie à ciel ouvert, qui devient, en 1988, la Commission canyon. Elle s'ouvre aux autres fédérations et permet une étroite collaboration de la FFS avec la Fédération Française de Canoë Kayak (FFCK) et la Fédération Française de la Montagne et de l'Escalade (FFME). La FFME crée une Commission canyon rattachée au Comité sportif alpinisme en 1991. La FFCK est moins impliquée dans le canyoning et ne possède pas de structure interne gérant cette activité. En 1996, la Commission canyon de la FFS est rebaptisée École Française de Canyon (EFC) et souhaite alors que le ministère de la Jeunesse et des Sports lui délègue l'organisation complète de l'activité canyoning. Mais elle retire sa demande de délégation le 26 mars 1996. Deborde (1996) montre qu'il est difficile d'obtenir une explication officielle de ce retrait. C'est la FFME qui obtient cette délégation en août 1997. Elle continue encore à collaborer avec les deux autres fédérations dans un souci de concertation et de représentation la plus large possible de la famille des canyonistes (Commission canyon de la FFME, 1999). Il faut encore préciser que la pratique du canyoning intéresse également la fédération des clubs alpins français (CAF), car ayant une vocation de multi-activité, elle propose à ses adhérents un large choix d'activités de montagne. Depuis 
les années 1990, la majorité de ses clubs a intégré le canyoning au sein de ses programmes.

Parallèlement à la délégation de l'organisation de l'activité à une fédération sportive, l'autre composante de l'institutionnalisation du canyoning a été la mise en place d'un Brevet d'État d'Éducateur Sportif (BEES). En effet, selon la loi du 16 juillet 1984 modifiée par celle du 13 juillet 1992, puis par celle du 6 juillet 2000, l'encadrement sportif contre rémunération est réservé aux titulaires d'un BEES. En ce qui concerne le canyoning, « la mise en place d'une qualification pour l'encadrement professionnel a suscité dès 1990 de longs et vifs débats dans la communauté sportive » (Pinguet, 1996, p. 44), notamment entre les guides de haute montagne, les accompagnateurs de moyenne montagne, les moniteurs d'escalade, de spéléologie et ceux de l'eau vive. L'activité étant revendiquée par des professionnels de filières concurrentes, c'est le ministère de la Jeunesse et des Sports qui a dû opérer un arbitrage serré entre les multiples intérêts en présence. Désormais, la réglementation définit clairement les professionnels en charge de l'activité (Barull, 1998). Depuis le 31 août 1996, seuls les BEES option escalade, spéléologie et alpinisme sont autorisés à encadrer l'activité. La formation est désormais intégrée et obligatoire dans chacun de ces diplômes à cordes. Les accompagnateurs de moyenne montagne ont la possibilité d'encadrer la randonnée aquatique, dans les canyons non verticaux et non aquatiques qui n'exigent pas pour la progression l'utilisation d'agrès. La situation des autres professionnels encadrant l'activité avant 1996 a été régularisée avec l'Attestation de Qualification d'Accompagnement (AQA).

\section{Le canyoning sur le site du Furon}

\section{Histoire des activités sur le site du Furon}

Le Furon est une rivière qui prend sa source sur le plateau du Vercors pour se jeter dans le Drac au niveau de la commune de Sassenage. Les premiers utilisateurs du canyon du Furon sont les pêcheurs. En effet, l'association de pêche de Sassenage existe depuis 1932. La partie basse du Furon est aussi fréquentée depuis longtemps par des promeneurs et des baigneurs. En 1954, un barrage EDF fut construit sur la commune d'Engins, en amont du canyon. Les premiers canyoneurs ont investi la partie basse du canyon dans les années 1980. Cette pratique a pris de l'essor et le nombre de pratiquants n'a cessé d'augmenter à partir de ce moment. Il y 
a une dizaine d'années, les professionnels du canyoning se sont rendus compte que la partie haute du Furon était intéressante pour la pratique. Depuis, la fréquentation de cette partie a considérablement augmenté.

\section{Présentation du canyon}

Le canyon du Furon se divise en trois parties distinctes :

- La partie haute, qui débute juste en aval du barrage EDF, est une course d'initiation, qui se fait dans la journée, avec de nombreux sauts et toboggans. Il est possible, sur une demi-journée, de ne faire que la fin de cette partie, appelée « Furon express ».

- La partie moyenne, d'une longueur de $1,5 \mathrm{~km}$, présente peu d'intérêt pour la pratique du canyoning, mais elle est beaucoup utilisée par les pêcheurs.

- La partie basse est une course d'initiation un peu plus difficile que la partie supérieure, avec de nombreux sauts et quelques descentes en rappel.

Certains pratiquants réalisent "l'intégrale du Furon », c'est-à-dire qu'ils enchaînent la partie haute et la partie basse. Dans ce cas-là, les pratiquants sortent du canyon et empruntent le chemin de berge le long de la partie moyenne.

\section{Fréquentation du canyon}

Selon une étude de fréquentation menée au sein du Parc du Vercors (Boudières, 2000), le Furon est le canyon le plus fréquenté du massif. Plus précisément, en juillet et août 2000, 2277 canyoneurs ont fréquenté la partie haute du Furon et 441 ont fréquenté la partie basse du canyon. Le taux de pratiquants encadrés par un professionnel ou exerçant dans le cadre de l'activité d'un club est très élevé dans la partie haute $(93 \%)$ et plus faible dans la partie basse (47\%). Ainsi, il est possible d'observer que des pratiquants exercent régulièrement en dehors de toute structure.

\section{Le risque de débordement du barrage EDF}

Le barrage EDF en amont du canyon est un barrage déversant qui ne possède pas de vannes pour effectuer des lâchers d'eau. L'électricité n'est donc pas produite au niveau de ce barrage mais à la centrale de Sassenage, en aval du canyon, par un système de conduites forcées. La 
centrale peut turbiner un débit de $2,10 \mathrm{~m}^{3} / \mathrm{s}$ et le débit restitué en aval du barrage est de $601 / \mathrm{s}$.

Il existe deux cas de débordement du barrage. Dans un premier cas, si le débit entrant est supérieur à $2,10 \mathrm{~m}^{3} / \mathrm{s}$, toute l'eau ne peut être turbinée par la centrale de Sassenage. Par conséquent, l'eau s'accumule progressivement dans le barrage et déborde lorsqu'il est plein. Ceci se produit principalement en période de fonte des neiges, de février à début mai, et en période de gros orages à l'automne, ou de pluies exceptionnelles le reste du temps. Dans ce cas, le débit du Furon dans la partie aval est aussi augmenté par la confluence des Cuves de Sassenage. Dans un deuxième cas, le risque de débordement est lié à une panne éventuelle de la turbine. L'eau, qui était turbinée par la centrale, remplit alors le barrage et celui-ci déborde lorsqu'il est plein. Le cas le plus dangereux se produit lorsque la centrale fonctionne à son maximum, c'est-à-dire qu'elle turbine $2 \mathrm{~m}^{3} / \mathrm{s}$; car, si la panne survient, ce sont $2 \mathrm{~m}^{3} / \mathrm{s}$ d'eau, auxquels s'ajoute le débit entrant, qui se déversent directement dans le Furon. Selon le directeur du groupement de Saint-Georges-de-Commier d'EDF qui exploite la centrale, l'arrêt des turbines est possible et il se produit au maximum quatre fois par an.

Le risque de débordement du barrage pèse fortement sur la sécurité des pêcheurs et des pratiquants du canyoning engagés dans le canyon. Cette question de la sécurité en aval des ouvrages EDF a été soulevée lors de la catastrophe du Drac en 1995, où plusieurs personnes ont trouvé la mort. Pour pallier ce risque, le préfet, à la suite du procès de cette affaire, a pris un arrêté le 9 juillet 1997 sur tous les cours d'eau de l'Isère en aval d'ouvrages EDF, y compris le Furon. Il en a interdit totalement l'accès.

\section{L'arrêté préfectoral n॰ 97-6974 du 29 octobre 1997}

À la suite la prise de cet arrêté, les pêcheurs appartenant à l'Association Agréée de Pêche et de Protection du Milieu Aquatique (AAPPMA) de Sassenage et les professionnels du canyoning appuyés par leurs syndicats se sont mobilisés contre cette réglementation qui leur interdisait l'accès au canyon du Furon. Les pêcheurs ont notamment organisé des manifestations et ont fait circuler des pétitions avant de solliciter l'intervention de la fédération départementale de la pêche de l'Isère.

À la suite de cette mobilisation, deux réunions pilotées par les maires ont eu lieu au cours du mois d'août 1997. Elles ont abouti au remplacement de l'arrêté préfectoral du 9 juillet 1997 par celui du 29 octobre 1997 
qui est encore en vigueur aujourd'hui. Il autorise, par dérogation, l'accès au lit du Furon à différentes catégories d'acteurs.

- Aux pratiquants du canyoning dans le cadre d'associations affiliées ou d'établissements agréés par la FFME ou la FFS, ou encadrés par un professionnel membre d'un syndicat. Pour que cette dérogation soit effective, la signature d'une convention entre EDF Énergie Alpes et les Comités départementaux des fédérations et des représentants des syndicats est nécessaire.

- Aux pêcheurs affiliés à une association de pêche agréée sous réserve de l'établissement d'une convention entre la fédération départementale de pêche et EDF Énergie Alpes. Les écoles de pêche sont exclues de cette dérogation.

L'accès est donc interdit aux pratiquants exerçant en dehors de toute structure.

En définitive, sur le canyon du Furon se pose le problème de l'accès au site. Pour le résoudre, la réglementation en vigueur impose la signature de conventions entre EDF et des acteurs institutionnels représentant les pratiquants du canyoning et les pêcheurs. Elle rend donc nécessaire la coopération entre ces différents acteurs. C'est justement l'objet de l'approche organisationnelle développée par Friedberg (1993, p. 11) que d'étudier les processus d'organisation «par lesquels sont stabilisés et coordonnés les comportements et les interactions stratégiques d'un certain nombre d'acteurs dont l'interdépendance rend la coopération indispensable, mais qui gardent tous un degré d'autonomie et continuent, pour toutes sortes de raisons, à poursuivre des intérêts divergents ». Les concepts développés par cette approche vont donc être utilisés pour tenter de comprendre comment sont stabilisées les interactions entre les différents acteurs concernés par le problème de l'accès au site.

Il convient de préciser que, sur le site du Furon, si les pratiquants du canyoning qui exercent en dehors de toute structure sont concernés par ce problème de l'accès au site, la réglementation en vigueur les exclut des négociations.

\section{Cadre théorique et problématique}

\section{La dynamique de l'action organisée}

L'approche de Friedberg (1993) se situe dans la continuité de l'analyse stratégique des organisations qu'il a développée avec Crozier (Crozier 
et Friedberg, 1977). Selon Friedberg (1993, p. 154), il n'y a «aucune différence de nature entre une organisation formelle et des formes plus diffuses d'action collective, tout au plus une différence de degré ». Ainsi, quelles que soient les caractéristiques du contexte dans lequel elle se déploie, "toute action collective est d'une certaine façon organisée » (p. 177). Comme pour l'étude des organisations, il s'agit d'étudier au cas par cas chaque contexte d'action particulier, afin de comprendre les caractéristiques de leur structuration.

\section{L'ordre local}

Dans cette approche, Friedberg (1993, p. 12) s'intéresse à la production des ordres locaux, "ordre par lequel les acteurs impliqués structurent leur coopération, gèrent leurs interdépendances et institutionnalisent leurs espaces d'action ». L'ordre local peut être défini comme « un espace de concurrence de jeux réglés entre acteurs mutuellement dépendants autour d'un problème qu'ils ne peuvent résoudre seuls, et pour la solution duquel ils ont besoin d'obtenir la coopération de partenaires qui sont aussi des concurrents potentiels » (Friedberg, 1993, p. 172). Cet ordre local ne peut exister qu'à deux conditions (Mounet, 2000) :

- une confrontation de plusieurs acteurs à un problème commun : au sens où l'entend Friedberg, le problème peut être un obstacle à surmonter, mais également un projet concernant différents acteurs ;

- une interdépendance stratégique des acteurs autour du problème ; Friedberg précise qu'il y a interdépendance entre les acteurs à partir du moment où l'on constate des régularités dans leurs interactions.

Il s'agit alors de traduire un problème en un réseau d'acteurs, concernés directement ou indirectement par le traitement de ce problème. Puis, il faut décrire et analyser la structure des relations entre ces acteurs pour comprendre en quoi ce réseau forme un ordre local. L'arbitraire, au moins initial, est du côté de l'analyste : il peut s'intéresser à tous les problèmes et essayer de les traduire en un système d'acteurs, pour peu qu'il parvienne à en démontrer l'existence.

Selon Friedberg (1993), l'acteur est empirique dans la mesure où son appartenance au contexte d'action est une question de recherche. En effet, au départ, rien ne permet de faire un tri entre les individus concernés par le problème dont il s'agit de comprendre la structuration sociale : ce n'est que le processus de recherche qui pourra permettre ce tri. Ainsi, il est possible de « découvrir que tel acteur, dont on pensait a priori qu'il jouait un rôle important et qu'il s'investissait fortement dans les interactions au sein 
du système, soit n'est pas un acteur du tout, soit l'est mais de façon très particulière et limitée » (Friedberg, 1993, p. 269). Un individu a donc le statut d'acteur dans un champ d'action dans la mesure où l'on peut montrer que son comportement contribue à structurer ce champ : « Exister en tant qu'acteur dans un champ signifie ipso facto participer à sa régulation » (Friedberg, 1993, p. 224).

Friedberg précise que les acteurs peuvent être individuels ou collectifs. Un acteur collectif est le porte-parole d'une organisation et représente les intérêts convergents des membres de cette organisation. Alors, "pour l'étude de certains problèmes, une centrale nucléaire ou le Club Med peuvent être considérés comme des acteurs relativement unifiés, sans que l'on soit obligé de rentrer dans le détail des systèmes d'acteurs. À d'autres moments, et pour d'autres questions, un tel démontage des acteurs composites (ou collectifs) peut, au contraire, s'avérer indispensable » (Friedberg, 1993, p. 244).

\section{Pouvoir et incertitude}

L'ordre local se construit autour d'un problème et rassemble tous les acteurs concernés par ce problème. Or tout problème, quel qu'il soit, comporte toujours une part d'incertitude. Les systèmes d'acteurs se structurent toujours autour des incertitudes naturelles ou objectives découlant des caractéristiques techniques, économiques ou autres des problèmes à résoudre. Au moins dans le court terme, ces incertitudes s'imposent comme une donnée aux acteurs. Mais rapidement, les jeux entre les acteurs redéfinissent les problèmes et restructurent les champs d'action (Friedberg, 1993). Les acteurs créent notamment des incertitudes artificielles qui permettent de contrecarrer les incertitudes naturelles et de rééquilibrer les marchandages entre les participants.

Or les incertitudes constituent en même temps la principale ressource des acteurs dans leurs négociations les uns avec les autres, car " ce qui est incertitude du point de vue des problèmes est pouvoir du point de vue des acteurs » (Friedberg, 1993, p. 250). Les rapports des acteurs s'inscrivent dans un champ inégalitaire, structuré par des relations de pouvoir. Celui-ci est conceptualisé comme une "relation d'échange négocié de comportements ». Friedberg précise que les relations de pouvoir ne sont pas seulement conflictuelles; " elles incluent toujours une dimension collusive dans la mesure où chaque acteur pour améliorer sa propre position cherche à réduire les possibilités de choix de ses partenaires et contribue à la stabilité de la relation » (p. 130). 


\section{Une problématique organisationnelle}

Sur le site du Furon, plusieurs acteurs sont en interaction stratégique autour du problème de l'accès au canyon, problème qu'ils ne peuvent résoudre seuls. L'approche organisationnelle va permettre de mettre en évidence la structure de relations et d'échanges entre cet ensemble d'acteurs. Elle va permettre de comprendre comment les acteurs se sont organisés pour résoudre ce problème.

L'analyse doit être menée à différents niveaux.

Dans un premier temps, il s'agit de délimiter le système d'acteurs $\mathrm{du}$ contexte d'action, c'est-à-dire faire un inventaire des acteurs concernés par le problème de l'accès au canyon du Furon. Mounet (2000) précise qu'il est nécessaire de s'intéresser au statut, aux ressources financières et matérielles et au rôle formel des acteurs dans le contexte d'action, ceci " en raison du manque d'information exhaustive sur les champs organisationnels étudiés » (p. 41).

Il est également utile de comprendre l'équilibre qui existe entre les acteurs pour caractériser le degré d'organisation du contexte d'action. Les relations entre les acteurs sont plus ou moins formalisées. Il peut exister des conventions ou des accords entre certains qui structurent le champ organisationnel. Mais, comme l'explique Friedberg (1993), le degré de formalisation seul ne suffit à caractériser le degré d'organisation. Il faut ajouter trois autres dimensions qui sont le degré de finalisation, le degré de conscience et le degré de délégation de la régulation.

Friedberg (1993, p. 225) précise qu'un système «n'est explicable qu'à partir de l'action qui l'institue et le ré-institue constamment ». Après avoir mis en évidence quels étaient les différents acteurs du système, il faut donc s'intéresser à leurs actions.

Selon Friedberg (1993, p. 259), les acteurs de tout contexte d'action ont « des intentions et des objectifs même s'ils ne réussissent pas souvent à les réaliser, ils sont capables de choix et ils poursuivent ce qu'ils considèrent être leurs intérêts ». Il faut donc s'intéresser aux objectifs et aux intérêts des acteurs, objectifs qui peuvent dépasser leur rôle formel. Souvent, si les acteurs veulent atteindre leurs objectifs, ils ont besoin d'obtenir la contribution d'autres acteurs. Ils peuvent alors coopérer même si leurs objectifs ne sont pas complètement convergents.

Friedberg (1993, p. 251) précise que le système de relations de pouvoir est « étayé sur les incertitudes inhérentes aux problèmes à résoudre ». 
Il convient donc de préciser quelles sont ces incertitudes qui constituent une ressource pour les acteurs dans leur négociation.

\section{Hypothèses de recherche}

Sur le site du Furon, des acteurs uniquement institutionnels sont en interaction stratégique autour du problème de l'accès au canyon.

Les pratiquants inorganisés ne font pas partie de l'ordre local, non parce qu'ils ne sont pas intéressés par le problème qui le sous-tend, mais parce qu'ils sont exclus des négociations par la réglementation. Ils ne sont donc pas en interaction stratégique avec les acteurs institutionnels. Cependant, ces pratiquants inorganisés sont bénéficiaires des accords négociés.

\section{Méthodologie}

Friedberg (1993, p. 294) explique que « devant la nature irréductiblement contingente du phénomène auquel elle s'intéresse, l'approche organisationnelle de l'action sociale ne peut s'engager que dans une analyse clinique et pour tout dire également et nécessairement contingente de la réalité des relations qui, dans un espace d'action particulier, se nouent entre les acteurs concernés ». Cette démarche, qui repose sur des méthodes cliniques et qualitatives, "part du vécu des acteurs pour reconstruire non pas la structure sociale générale, mais la logique et les propriétés particulières d'un ordre local » (p. 304).

\section{Les entretiens semi-directifs}

\section{Le guide d'entretien}

Dans la démarche adoptée, l'entretien semi-directif tient une place privilégiée. Un guide d'entretien a été élaboré dont les indicateurs portent sur :

- l'analyse systémique : l'inventaire des acteurs et le degré d'organisation du système ;

- l'analyse stratégique : les objectifs et les moyens mis en œuvre par les acteurs et leurs relations au sein de l'ordre local.

\section{Les acteurs interrogés}

Les entretiens semi-directifs se sont déroulés avec les acteurs institutionnels concernés par le problème et ayant un rôle dans la structuration du contexte d'action. Il convient de préciser que chaque institution est consi- 
dérée comme un acteur collectif relativement unifié (Friedberg, 1993), et n'est pris en compte qu'un porte-parole pertinent de chacune d'elles, même s'il " est acquis que chaque acteur individuel a ses propres objectifs qui peuvent être distincts de ceux de l'organisation à laquelle il appartient » (Mounet, 2000, p. 187).

Les acteurs interrogés sont les suivants.

- Les deux sections locales des syndicats de professionnels du canyoning qui représentent l'ensemble des professionnels du Vercors, dans le but de défendre leurs intérêts. Il s'agit de la section locale Drôme-Isère du Syndicat National des Professionnels Spéléo-Canyon (SNPSC) et la compagnie des guides du Dauphiné, représentées par leurs présidents.

- Le CAF Isère, représenté par le responsable de la section descente de canyon. Ce club de 3000 adhérents propose des sorties de canyoning sur le Furon depuis 1996.

- L'Association Agréée de Pêche et de Protection du Milieu Aquatique (AAPPMA) de Sassenage, représentée par son président. C'est une association réciprocitaire avec les autres AAPPMA du département, c'est-à-dire que ses structures ouvrent réciproquement à leurs adhérents respectifs l'accès aux lots de pêche qu'elles gèrent.

- Les maires des communes d'Engins et de Sassenage sur le territoire desquelles se trouve le canyon du Furon.

- EDF Énergie Alpes, groupe d'exploitation hydraulique Drac, représenté par son directeur. L'entreprise est propriétaire du barrage d'Engins et de la centrale de Sassenage.

- Le comité départemental Isère de la FFME, représenté par son président.

- La fédération départementale de l'Isère de pêche et de protection du milieu aquatique, représentée par son président. Cette fédération fait partie du groupement réciprocitaire interdépartemental le Club halieutique, qui regroupe 35 départements.

- L'office national des forêts (ONF) de Sassenage représentée par son technicien. Dans le cadre d'une convention qui lie l'ONF à la mairie de Sassenage, le rôle de l'ONF est de faire appliquer les arrêtés préfectoraux et municipaux.

- Le PNRV représenté par le chargé de mission «sports de nature ». Les entretiens se sont déroulés lors des mois de mars et avril 2000. 


\section{L’analyse des données}

Pour analyser les données recueillies par entretiens semi-directifs, la méthode retenue a été celle de l'analyse de contenu. Plus précisément, l'analyse catégorielle a été privilégiée. "Elle fonctionne par opérations de découpage du texte en unités puis classification de ces unités en catégories selon des regroupements analogiques » (Bardin, 1977, p. 155). Parmi les différentes catégorisations, c'est l'investigation des thèmes qui a été choisie. Berelson (1971, p. 152) définit le thème comme « une affirmation sur un sujet, c'est-à-dire une phrase, ou une phrase composée, habituellement un résumé ou une phrase condensée, sous laquelle un vaste ensemble de formulations singulières peuvent être affectées ". Bardin (1977, p. 105) explique que « faire une analyse thématique consiste à repérer des noyaux de sens qui composent la communication et dont la présence ou la fréquence d'apparition pourront signifier quelque chose pour l'objectif analytique choisi ».

\section{L'analyse de documents}

Une analyse de documents a permis de compléter les données recueillies par entretien. Ainsi, les textes des conventions, recueillis auprès des acteurs lors des entretiens, ont été analysés. L'objectif de cette analyse a été de déceler les zones d'incertitude contenues dans ces textes.

\section{L'observation directe}

Une observation directe a eu lieu sur le site du Furon au cours des mois de juillet et août 2000, dans le but de connaître les différents usages du site, et la répartition spatiale des pratiquants.

\section{Résultats}

\section{L'établissement de conventions}

À la suite de la modification de l'arrêté préfectoral, des négociations ont eu lieu entre EDF et les acteurs institutionnels représentant les pratiquants du canyoning et les pêcheurs.

D'une part, pour permettre l'accès du canyon aux pêcheurs, c'est la fédération départementale de la pêche qui représente les intérêts de l'en- 
semble des pêcheurs, qui a négocié directement avec EDF Énergie Alpes GEH Drac. L'AAPPMA de Sassenage n'a donc pas participé aux négociations. Une convention a été signée entre EDF et la fédération, le 13 mars 1998. Elle définit les informations communiquées par EDF sur ses ouvrages. La fédération est chargée de diffuser ces informations aux pêcheurs en leur remettant, lors de l'achat du permis de pêche, un dépliant dont la lecture est obligatoire. Cette convention est renouvelable chaque année et elle a été reconduite pour la saison de pêche de l'année 2000.

D'autre part, pour permettre l'accès du canyon aux canyoneurs, des négociations ont eu lieu entre EDF Énergie Alpes et les représentants des pratiquants du canyoning, c'est-à-dire le Comité départemental de la FFME, les deux syndicats des professionnels, ainsi que le CAF Isère. Toutefois, EDF souhaitait n'avoir qu'un seul interlocuteur. Étant donné que la FFME est la fédération délégataire pour l'organisation du canyoning, c'est le comité départemental de la FFME qui a signé une convention avec EDF le 19 juin 1998. Le comité représente donc l'ensemble des organisations reconnues pour la pratique du canyoning sur le Furon. La convention définit les informations délivrées par EDF sur le fonctionnement des ouvrages hydro-électriques et sur la mesure du débit entrant dans la retenue d'Engins. La FFME y délivre des informations sur les conditions de la pratique du canyoning dans le Furon. Dans le cadre de cette convention, le rôle des communes d'Engins et de Sassenage est de « faciliter la mise en place de moyens d'information adaptés pour permettre l'application de la convention ». Cette convention était d'une durée d'un an. Elle est donc caduque depuis le 19 juin 1999, et depuis cette date, la dérogation pour la pratique du canyoning dans le Furon n'est plus valable. Une nouvelle convention est en cours d'élaboration.

Ainsi EDF, en signant des conventions, choisit de prendre en compte les autres utilisateurs. S'il accepte de négocier avec les autres acteurs, c'est que cela lui permet de poursuivre un objectif que l'entreprise s'est fixé au niveau national : améliorer son image auprès du grand public. Comme l'affirme le directeur du GEH Drac, « je crois qu'on a été pas mal sensibilisé au fait qu'EDF n'a plus les rivières pour lui. Enfin, il ne les a jamais eues pour lui ; mais on a été sensibilisé au fait qu'il fallait prendre en compte les autres acteurs de l'eau, les autres partenaires de l'eau et voir comment est-ce que l'on pouvait exploiter nos installations tout en laissant une place aux autres. » La rivière, lieu dédié à la production d'électricité, est donc devenue un espace de loisir.

En annexe de la convention signée avec le comité départemental de la FFME, EDF délivre des informations relatives au temps de remplissage 
du barrage en fonction du niveau d'eau et du débit entrant dans la retenue, en cas d'arrêt de la turbine. Les pratiquants du canyoning, en consultant ces données peuvent calculer le temps dont ils disposent pour faire leur course en sécurité. Ainsi, EDF réduit l'incertitude naturelle liée au risque de débordement du barrage. En revanche, cette convention comporte des zones d'incertitude : elle n'explique pas comment les pratiquants licenciés dans un autre département ont accès à cette information. Elle n'explique pas non plus quels sont les moyens d'information adaptés que les communes doivent mettre en place pour faciliter l'application de la convention. Finalement, même si la formulation de règles formelles a permis de réduire une incertitude naturelle, ces règles laissent encore des zones d'incertitude. Les incertitudes liées au problème n'ont donc pas disparu, mais elles ont été déplacées par les acteurs. C'est pour cela que Friedberg (1993, p. 238) explique que "la structure des incertitudes naturelles et artificielles, autour desquelles se sont cristallisés les jeux, est soumise à $d$ 'incessants changements ».

Les fédérations, en signant des conventions avec EDF, permettent aux professionnels membres d'un syndicat, aux pratiquants du CAF Isère et aux pêcheurs de l'AAPPMA de Sassenage d'atteindre leur objectif qui est de pouvoir accéder au Furon. Elles apportent donc une solution à leurs licenciés, et elles augmentent la pertinence de leur possibilité d'action (Friedberg, 1993). Il est intéressant de noter que la pertinence du comité départemental de la FFME provient des contraintes de la préstructuration. En effet, depuis la loi n 84-610 du 16 juillet 1984, le ministère de la Jeunesse et des Sports délègue le monopole de l'organisation d'une pratique sportive à une fédération. La FFME, ayant reçu cette délégation pour la pratique du canyoning, obtient ainsi une légitimité auprès des acteurs institutionnels. Et alors que, sur le site du Furon, les principaux utilisateurs sont le CAF Isère et les professionnels, c'est le comité départemental de la FFME, qui, fort de cette légitimité, a été reconnu par EDF comme le seul interlocuteur pour la signature de la convention. Cependant, la pertinence du comité départemental est moins importante que celle de la fédération départementale de pêche puisque la convention qu'il a signée est caduque. Comme sa durée ne peut excéder une année, le comité est obligé de renouveler ses négociations avec EDF tous les ans. Au contraire, l'entreprise a accepté de signer une convention à tacite reconduction avec la fédération départementale de la pêche. La pratique de la pêche est donc mieux reconnue que celle du canyoning ; elle a plus de légitimité aux yeux d'un acteur tel qu'EDF. 
Il convient de préciser que le PNRV n'a pas été impliqué dans les négociations car « la convention a été travaillée sans le Parc : à l'époque, en effet, on n'était pas encore engagé dans la démarche de schéma d'organisation des activités de plein air. Cela s'est fait juste au début où nous, on démarrait notre action d'animation. Donc on n'était pas impliqué du tout sur cette histoire de convention sur le Furon. »

En définitive, les différents acteurs concernés par le problème de l'accès au canyon du Furon ne pouvant résoudre ce problème seuls, ont été amenés à négocier les uns avec les autres pour trouver une solution satisfaisante. Le résultat de ces négociations a été l'établissement de conventions qui, comme toutes les règles, correspondent à des compromis, mais surtout, " ont toujours un rôle de régulation des rapports de coopérations conflictuelles" (Friedberg, 1993, p. 180). Les règles sont donc toujours ambivalentes : « correspondant à des contraintes, elles cristallisent et reflètent aussi toujours un minimum de collusion en faveur de la stabilité de la relation de marchandage et autour du compromis auquel celle-ci a donné naissance ». Ainsi, l'établissement de conventions a permis de stabiliser les interactions stratégiques des acteurs.

Friedberg (1993) précise que la formalisation de la régulation va de pair avec une certaine centralisation. "De même elle implique une certaine finalisation de la régulation et surtout un minimum de conscience et d'acceptation de la part des participants de leurs interdépendances et de leur régulation » (p. 156). Ainsi, dans le contexte d'action, une partie de la régulation a été déléguée aux trois acteurs que sont EDF et les deux fédérations. Les mécanismes de régulation sont articulés autour d'un but qui est de rouvrir le site du Furon, tout en permettant à EDF de mettre sa responsabilité à l'abri. Les acteurs qui ont participé aux négociations ont un degré de conscience élevé de ce but.

\section{Des intérêts et des enjeux}

Selon Friedberg (1993), même si la règle finit par figer un compromis qui peut être analysé comme un accord qui structure les échanges, "son existence ne signifie pas pour autant la fin des jeux de pouvoir » (p. 283). Chaque acteur poursuit ce qu'il considère être ses intérêts.

Dans l'ordre local, les pêcheurs et les canyoneurs ont des intérêts convergents. Le CAF souhaite que tous les usagers puissent accéder au Furon : «nous, on est là aussi pour défendre les intérêts des usagers et pas uniquement ceux des cafistes. [...] Nous, on est licenciés donc nous, on y a accès. Mais on défend aussi le commun vulgus [sic] qui, lui, n'est 
pas licencié et qui a droit tout autant que nous à aller dans le Furon; ou tout simplement la famille qui fait son pique-nique et qui veut se tremper avec des enfants. » Le président de la section locale du SNPSC explique que "nous, les professionnels, on va d'abord se battre pour que l'accès soit autorisé aux professionnels avant de se battre pour tous les pratiquants. [...] Mais je défends aussi la liberté de chacun. Je suis pour (le fait) qu'il y ait le moins possible de convention et le plus possible de liberté de circuler. » Les fédérations affirment également vouloir obtenir une liberté d'accès au site pour tous. Pour le président de la fédération départementale de la pêche, " le but ultime, il est pour les usagers, que ce soient des sportifs ou des pêcheurs ou des promeneurs ; le but ultime est bien qu'il n'y ait plus besoin de faire de convention pour y aller, et à la limite qu'il n'y ait plus d'arrêté préfectoral, plus d'interdiction. » Il en est de même pour le comité départemental de la FFME, selon lequel « il ne devrait pas y avoir de convention. Enfin, nous, c'est ce que l'on souhaite. »

Les syndicats de professionnels et le CAF Isère s'accordent sur le fait qu'une sécurisation des ouvrages EDF permettrait à tous d'accéder au canyon. Selon ces acteurs, " s'il y a un danger, c'est à EDF de faire le nécessaire pour que les gens soient avertis du danger. [...] Ils pourraient mettre en place un système innovant : une petite coloration ou un signal sonore. » Le président de la section locale du SNPSC explique « qu'il y a une solution pour éviter les débordements du barrage : c'est d'avoir un système de bascule, c'est-à-dire un bi-passe. » Il s'agit d'une dérivation parallèle installée au niveau de l'usine hydroélectrique. "Si la turbine s'arrête, la vanne se met en route et évite simplement la turbine. À ce moment, l'eau ne déborde plus au niveau du barrage, et cela ne pose pas de problème car elle ressort directement après la turbine. » Afin de pouvoir négocier directement avec EDF, le président de la section locale du SNPSC a participé à différentes réunions sur la sécurité des zones situées à l'aval des ouvrages hydrauliques, organisées en préfecture au cours de l'année 1999. Ainsi, même si c'est le comité départemental de la FFME qui représente tous les organismes reconnus pour la pratique du canyoning, la section locale du SNPSC conserve une capacité d'action dans l'ordre local. Pour avoir plus de poids dans la négociation avec EDF, cet acteur s'est allié avec la fédération départementale de la pêche car ils se sont rendus compte qu'ils avaient les mêmes intérêts sur le site du Furon. Il est intéressant de noter cette coopération car, sur de nombreux sites où pêcheurs et canyoneurs sont présents, leurs relations sont conflictuelles du fait qu'ils doivent se partager la rivière. Cependant, les ressources de ces acteurs pour négocier avec EDF sont limitées, car, 
comme le déclare le président de la section locale du SNPSC, « c'est un peu le pot de fer contre le pot de terre. [...] Contrairement à EDF, nous, on ne se déplace pas avec des conseillers juridiques pour les lois qui sortent et les réglementations. »

Par ailleurs, dans l'ordre local, le Parc du Vercors, même s'il n'a pas fait partie des négociations pour l'élaboration de la convention permettant la pratique du canyoning sur le Furon, essaie de se tenir informé de la situation et développe pour cela une collaboration avec les syndicats des professionnels. Comme l'explique le chargé de mission sports de nature, " même si on n'était pas beaucoup impliqué dans la démarche au départ avec les conventionnements, on a maintenant plus d'information, on a des retours avec les professionnels pour faire vivre cette convention ». L'enjeu pour lui est de pouvoir gérer les activités de nature alors qu'il n'a pas de capacité réglementaire. Pour cela, il a besoin de connaître précisément l'ordre local, pour avoir la connaissance des règles formelles et des équilibres stratégiques. Cette connaissance va notamment lui permettre de réduire une zone d'incertitude présente dans le texte de la convention signée entre EDF et le comité départemental de la FFME, et augmenter ainsi sa pertinence dans l'ordre local. En effet, il faut comprendre que le panneau d'information sur les risques liés à l'exploitation des ouvrages EDF, destiné aux canyoneurs, est installé au niveau du barrage d'Engins. Or l'observation a montré que les pratiquants du canyoning qui fréquentent la partie haute accèdent au canyon à un kilomètre en aval du barrage. Ceux qui fréquentent la partie basse débutent leur course sur la commune de Sassenage. Les pratiquants qui ne connaissent pas l'existence de ce panneau n'ont donc pas accès à cette information, car ils ne passent pas devant. Pour réduire cette incertitude, le PNRV prévoit d'installer des panneaux d'information précisant aux pratiquants d'aller vérifier les modalités de pratique affichées au niveau du barrage d'Engins avant de s'engager dans le canyon. Ces panneaux seront installés au niveau des différents parkings où stationnent les pratiquants du canyoning. Ainsi, le Parc « informe, pour qu' on sache que la convention existe ; alors que ce n'était pas le cas jusqu'à maintenant ». Cette intervention du Parc lui permet d'exister dans l'ordre local.

De plus, la connaissance précise de l'ordre local est aussi celle des acteurs pertinents. Cette connaissance lui permettra de savoir avec quels acteurs il est intéressant pour lui de développer un partenariat afin de devenir pertinent dans l'ordre local. Sur le site du Furon, ce ne sont pas les syndicats des professionnels qui sont pertinents mais le comité départemental de la FFME. C'est donc avec cet acteur que le PNRV doit entamer 
des négociations s'il veut augmenter sa pertinence. C'est pour cette raison que si, pour le moment, le PNRV n'a aucune relation avec le comité, il aimerait les rencontrer " pour savoir un petit peu quelles pistes de travail on pourrait élaborer ensemble, et surtout quels types de contacts on pourrait avoir de façon un peu plus pérenne sur le Furon, mais aussi, pourquoi pas, sur l'ensemble des sites du Vercors ».

Enfin, en ce qui concerne les communes, le maire d'Engins n'a aucun objectif particulier concernant le canyoning. Cet acteur n'a aucune relation avec les autres acteurs. Il déclare : "Quand ça se passe bien, on ne voit personne. Il y a juste eu des négociations avec le préfet au moment de l'arrêté, mais après tout s'est réglé par convention. » Le maire de Sassenage en revanche a des objectifs qui dépassent son rôle prévu par la convention signée entre EDF et le comité départemental de la FFME. Il déclare que « le premier souci que je dois avoir, c'est le problème de la sécurité. Et bien entendu, cela touche aussi bien les gens qui vont se promener que ceux qui font des activités comme la pêche ou le canyoning. Et mon rôle est de faire en sorte qu'il y ait le moins d'imprudences possibles de commises. Il faut donc faire de la communication, il faut faire de l'information. [...] On a fait des panneaux placés à différents endroits pour donner les informations nécessaires par rapport au Furon ; pour dire : "C'est un torrent, il peut y avoir tel événement, tel autre événement, etc." » Ainsi, les moyens d'information que la commune a mis en place ne sont pas uniquement destinés aux canyoneurs mais à tous les utilisateurs du site. Pour réaliser ces panneaux, le maire de Sassenage a participé aux différentes réunions organisées en préfecture sur la sécurité des zones situées à l'aval des ouvrages hydrauliques, et a donc pu coopérer avec EDF : «On a beaucoup travaillé avec EDF jusqu'au mois de juillet dernier, pour mettre en valeur une espèce de règle de conduite entre les différents partenaires. »

En définitive, sur le site du Furon, EDF, la section locale du SNPSC, la Compagnie des guides du Dauphiné, le CAF Isère, le comité départemental de la FFME, la fédération département de la pêche, et la commune de Sassenage sont en interaction stratégique autour du problème de l'accès au canyon. Même s'ils gardent tous un degré d'autonomie et continuent à poursuivre des intérêts divergents, leurs comportements et leurs interactions stratégiques sont stabilisés et coordonnées sous la forme d'un ordre local. Les acteurs de cet ordre local sont uniquement des acteurs institutionnels. La première hypothèse est donc vérifiée. Pour le moment, le PNRV ne fait pas partie de cet ordre local car il n'a pas encore engagé d'action pour se rendre pertinent auprès des autres acteurs. 


\section{Le contournement de la règle}

De même, les baigneurs et les pratiquants du canyoning exerçant en dehors de toute structure sont absents de cet ordre local, étant donné qu'ils n'ont pas pris part dans les négociations pour l'élaboration des conventions, ni aux réunions organisées en préfecture. Ils ne sont pas en interaction stratégique avec les autres acteurs et il est impossible de les considérer comme des acteurs dans l'ordre local car, selon Friedberg (1993), un individu a un statut d'acteur dans un contexte d'action " dans la mesure où l'on peut montrer que son comportement contribue à structurer ce champ » (p. 209). Or les pratiquants non organisés et les baigneurs n'ont pas de poids dans le contexte d'action puisqu'ils ne se sont pas regroupés en acteurs collectifs autour d'intérêts communs. Ils n'ont donc pas de capacité d'action en tant que groupe pour pouvoir négocier avec les autres acteurs.

Cependant, ils sont à prendre en compte car ils peuvent influencer les négociateurs. En effet, les fédérations, les syndicats des professionnels et le CAF Isère prennent en compte ces usagers lorsqu'ils défendent l'accès au site pour tous. Ainsi, certains acteurs de l'ordre local représentent les inorganisés, mais c'est unilatéral dans la mesure où ils n'ont pas $\mathrm{d}$ 'interactions avec eux. La deuxième hypothèse n'est donc vérifiée qu'en partie, puisque s'il est possible d'affirmer que les pratiquants inorganisés ne sont pas en interaction stratégique avec les acteurs institutionnels, en revanche, ils ne sont pas bénéficiaires des accords négociés puisque l'accès au site leur est toujours interdit.

À ce niveau, il convient de préciser que, même si l'interdiction d'accès persiste pour ces acteurs, les résultats de l'étude de fréquentation (Boudières, 2000) ont montré que des pratiquants exerçant en dehors de toute structure continuaient de fréquenter le canyon. Et les observations sur le terrain ont mis en évidence la présence de baigneurs à l'arrivée de la partie basse du canyon. Ils contournent donc la règle. Cela s'explique par le fait que la mise en application de l'arrêté préfectoral est difficile. Comme l'explique le technicien de l'ONF de Sassenage chargé de le faire appliquer : "On a essayé de mettre en application l'arrêté préfectoral. Ce n'est pas possible. Les contrôles, on les a faits, mais on a vu que c'était irréalisable. C'est tout simple : l'arrêté préfectoral spécifie que la baignade est interdite. Alors quand est-ce qu'on se baigne, quand est-ce qu'on fait du canyoning? Vous pouvez me le dire vous? C'est une bonne question la différence entre la baignade et le canyoning. Et en plus de cela, l'arrêté préfectoral spécifiait que les gens qui font du canyoning 
doivent être accompagnés par un professionnel ou licenciés à une fédération. Alors on arrêtait les gens ; ils nous disaient :

«- Ah! Bah! Oui, je suis licencié.

- Mais elle est où votre licence?

- Elle est dans ma voiture ; elle est là haut.

On fait comment, nous ? Donc c'est irréalisable. »

Il faut également savoir que le seul panneau indiquant que « la descente de canyon est une activité réglementée par Arrêté Préfectoral dans ce site » est placé au niveau du barrage d'Engins. Or, comme cela a déjà été signalé plus haut dans le texte, les observations sur le terrain ont montré que les pratiquants du canyoning qui fréquentent la partie haute accèdent au canyon à un kilomètre en aval du barrage. Ceux qui fréquentent la partie basse débutent leur course sur la commune de Sassenage. Les pratiquants inorganisés qui ne connaissent pas le site n'ont donc pas accès à cette information, car ils ne passent pas devant le panneau.

Finalement, l'arrêté préfectoral comporte sa propre transgression dans la mesure où il est illégal. En effet, il fait la distinction entre plusieurs catégories de personnes et porte ainsi atteinte à la liberté d'aller et de venir, "qui est un des aspects de la liberté individuelle et qui compte au nombre des principes fondamentaux reconnus par les lois de la République » (Pinguet, 2000, p. 86). Il est alors possible d'affirmer que les pratiquants inorganisés sont bénéficiaires des accords négociés. La deuxième hypothèse est finalement vérifiée.

\section{Conclusion}

Ces résultats mettent en évidence la difficile gestion d'une activité sportive de nature telle que le canyoning. En effet, pour cette activité, il n'y a pas de gestionnaire désigné si ce n'est la fédération délégataire ; mais celle-ci ne regroupe pas tous les pratiquants. Et, en attendant la mise en place des futures commissions départementales des espaces sites et itinéraires relatifs aux sports de nature, il n'y a pour le moment pas de textes clairs qui réglementent la pratique de l'activité. Alors, en l'absence d'une régulation globale, la gestion s'organise sur chaque site de pratique en fonction des contraintes locales et des intérêts des acteurs en présence. 
Sur le site du Furon, la réglementation en vigueur impose à EDF de signer des conventions avec des acteurs institutionnels représentant les pratiquants. Concernant la pratique du canyoning, EDF a choisi de signer une convention avec le comité départemental de la FFME plutôt qu'avec les syndicats des professionnels. L'entreprise s'est donc tournée vers l'acteur qui représente la modalité d'institutionnalisation de l'activité qui lui paraissait la plus légitime. Le comité départemental s'est donc trouvé impliqué dans la gestion du canyoning sur le Furon, alors qu'il ne s'est positionné sur aucun autre site dans le département. C'est la raison pour laquelle il a un degré d'implication très faible sur le Furon. Les maires ne sont pas non plus des acteurs pertinents dans la gestion de l'activité car le cours d'eau se situe sur le territoire de deux communes; d'où la nécessité de trouver un gestionnaire qui se situe à l'échelon intercommunal. Le PNRV, structure intercommunale, propose justement une gestion de l'activité à l'échelle de son territoire. Cependant, sur le site du Furon, il n'est pas pertinent dans la mesure où il n'a pas encore engagé d'actions et doit donc trouver un moyen de s'engager dans le « jeu » avec les acteurs pertinents de l'ordre local.

En définitive, la gestion de l'activité canyoning sur le Furon se résume en une " gestion institutionnelle » qui couvre EDF du point de vue de sa responsabilité. Cette gestion revient à un contournement de l'interdiction sécuritaire dans le sens où des pratiquants inorganisés continuent de fréquenter le site. Ainsi, la tentative d'élimination illégale d'une catégorie de pratiquants par une réglementation sécuritaire aboutit à rétablir le libre accès de fait. Finalement, les sports de nature, généralement considérés comme "libres " par opposition à l'ensemble des sports codifiés (Augustin, 1994 ; Bernardet, 1991 ; Dupuis, 1991, Lacroix, 1994 ; Loret, 1995 ; Pociello, 1995), sont amenés à basculer dans l'organisation lorsqu'ils se massifient (Vigarello, 1981). Et à ce moment-là, leur encadrement réglementaire pose un tel problème qu'ils retrouvent finalement leurs caractéristiques d'origine par manque de capacité réglementaire à faire appliquer des décisions qui méconnaissent les réalités de leur pratique et les bases des libertés fondamentales prévues par la Constitution. 


\section{Bibliographie}

ALEXIS, E. (1997). Canot gonflable et néoprène. Info Canyon, 1, 3-6.

Agence touristique départementale de la Savoie (1997). Plan touristique départemental. Chambéry : Conseil Général de la Savoie.

Augustin, J.-P. (1994). Surf Atlantique, les territoires de l'éphémère. Talence: MSH Aquitaine.

BARDIN, L. (1977). L'analyse de contenu. Paris : PUF.

BARRul, R. (1998). Lexique du cadre institutionnel et réglementaire des Activités Physiques et Sportives. Paris : INSEP publications, $3^{\mathrm{e}}$ édition.

BERELSON, B. (1971). Content analysis in communication research. New York : Hafner.

BERNARDET, P. (1991). Des APPN aux APE : le rapport à l'environnement comme caractère générique. In Actes des Deuxièmes Assises des Activités Physiques de Pleine Nature (p. 404-413). Université Paul Sabatier, URF STAPS, Toulouse, 17-19 octobre 1991.

BOUDiÈRES, V. (2000). Résultats des comptages de l'étude sur les canyons du Vercors. Mémoire de stage de première année de l'IUP Loisir, Environnement, Sport et Tourisme, Université Joseph Fourier, Grenoble.

Commission canyon de la FFME (1999). Descente de canyon. Manuel de référence. Paris : Mercier.

Crozier, M., \& FriedberG, E. (1977). L'acteur et le système. Les contraintes de l'action collective. Paris : Le Seuil.

DEBORDE, C. (1996). L'offre française des parcours de canyoning : d'une caractérisation des parcours aux problèmes de gestion et d'aménagement de l'activité. Mémoire de maîtrise de l'Institut de Géographie Alpine non publié, Université Joseph Fourier, Grenoble.

DuPuIs, J. (1991). Une APPN, c'est quoi au juste ? In Actes des Deuxièmes Assises des Activités Physiques de Pleine Nature (p. 394-403). Université Paul Sabatier, UFR STAPS, Toulouse, 17-19 octobre.

Fédération des Parcs Naturels régionaux de France (2000). Les Parcs naturels régionaux : centrale argumentaire. Paris : Syros.

FRIEDBERG, E. (1993). Le pouvoir et la règle. Dynamiques de l'action organisée. Paris: Le Seuil.

GARRIGUES, J. (1988). Évolution de la pratique sportive des français de 1967 à 1984. Paris : INSEE.

IRLINGER, P., LOUVEAU, C., \& METOUdI, M. (1987). Les pratiques sportives des Français. Paris : INSEP.

LACROIX, G. (1984). Les activités de pleine nature et le thème de la glisse. Enjeux institutionnels, économiques et culturels : l'exemple de la planche à voile. Thèse de doctorat en sciences sociales, Université Paris VII, Paris. 
MOUNET, J.-P. (2000). Les activités sportives de nature en France: contraintes globales, flou organisationnel et stratégie d'acteurs. DHDR en Sciences et Techniques des Activités Physiques et Sportives, Université Joseph Fourier, Grenoble.

Peseux, J.-Y., SAgaert, L., Mounet, J.-P., \& Delaye, D. (1999). Pratique d'activités sportives et compatibilité avec la préservation des milieux naturels. Enquête auprès des Parcs naturels régionaux de France. Paris : Fédération des Parcs naturels régionaux de France et Ministère de la Jeunesse et des Sports.

PINGUET, A. (1996). La réglementation étatique du canyoning. Cahiers du CSSM, 6, 44-50.

PINGUET, A. (2000). Libres propos sur le cadre juridique des sites, supports d'activités physiques de pleine nature. Montagnes Méditerranéennes, 11, 85-91.

PoCIELlo, C. (1995). Les cultures sportives. Paris : PUF.

POUQUET, L. (1994). Le comportement des consommateurs d'articles de sport. Paris : CREDOC.

Rigolet, M.F. (1995). Aventure de plein air, nature à ciel ouvert, culture des loisirs verts... Un triptyque utopique - la pratique du canyoning et son intégration à la vie locale : le cas du Parc national des Cévennes. Mémoire de Maîtrise de l'Institut de Géographie Alpine non publié, Université Joseph Fourier, Grenoble.

VigARELLO, G. (1981). D'une nature... l'autre : les paradoxes du nouveau retour. In C. Pociello (Éd.) Sports et société (p. 239-247). Paris : Vigot. 